\title{
الأفعال الكلامية الإنجازية في حوار فيلم "قيامة أرطفرل" الحلقة الأولى
}

\author{
Syamsi Setiadi ${ }^{1}$, Muhammad Kamal ${ }^{2}$, Annisa Ayu Rahmah ${ }^{3}$ \\ Universitas Negeri Jakarta \\ ${ }^{1 *}$ syamsi.setiadi@unj.ac.id, ${ }^{2}$ m.kamal.ah@unj.ac.id
}

\begin{abstract}
This study aims to obtain a clear description of the form and function of illocutionary speech acts in the dialogue of the film "Qiyamatu Ertugrul" episode 1. The method used in this research is descriptive qualitative method. The research instrument is the researcher herself is assisted by a classification table which contains the types of illocutionary speech acts and their fusions. Thus the researcher got the results that illocutionary speech acts in the dialogue of the film "Qiyamatu Ertugrul" episode 1, are as follows: 19 assertive speech acts with a percentage of $21 \%$ consisting of a statement function of 16 utterances and 3 explanations of 3 speeches. Commissive speech acts were 8 utterances with a percentage of $8 \%$ consisting of the promise function of 6 utterances and threats of 2 utterances. The directive speech acts were 47 speeches with a percentage of $53 \%$ consisting of the function of forcing 1 speech, giving advice as much as 1 speech, forcing 2 speeches, praying for 2 speeches, asking for 3 speeches, offering as many as 3 speeches, begging as much as 5 utterances, and ordered as many as 30 speeches. Expressive speech acts were 15 utterances with a percentage of $17 \%$ consisting of praising 6 utterances, thanking 5 utterances and congratulating 4 utterances. There are also declarative speech acts that are not found in the dialogue of this film.
\end{abstract}

\section{الكلمة الأساسية: الأفعال الكلامية الإنجازية، قيامة أرطفرل}

$$
\begin{aligned}
& \text { ففعل قولي (lokusi) وهو فعل التلفظ بجملة مع شرط }
\end{aligned}
$$

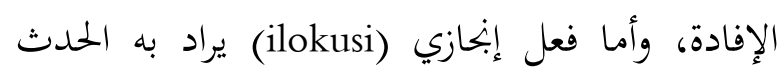

$$
\begin{aligned}
& \text { الذي يقصده المتكلم بالجملة كالأمر والتحذير، وفعل } \\
& \text { تأثيري (perlokusi) هو التأثير الذب يوقعه الحدث } \\
& \text { اللساني في المخاطب أو المتلقي كطاعة الأمر وتقبل } \\
& \text { النصيحة. } \\
& \text { بالإضافة إلى وجودها في الاتصال اليومي، } \\
& \text { يمكن أيضًا التوافر على أفعال الكالام في الأعمال } \\
& \text { الأدبية و من بينها في الأفلام. ورسائل الاتصال في } \\
& \text { فيلم أن تحقق فعل كلام، أي من خحلال المحادثات التي }
\end{aligned}
$$

${ }^{2}$ J. L Austin, How to Do Things with Words (London: Oxford University Press, 1962). H. 12
المقدمة

$$
\begin{aligned}
& \text { اللغة هي أداة للتفاعل الإجتماعي أو أداة } \\
& \text { للتواصل البشري.1 فيمكن أن يرتبط استخدام اللغة من } \\
& \text { قبل البشر بالمتحدثين والخاطبون، وبهذا سيشكل أفعالا } \\
& \text { في الكالام أو يمكن أن يسمى عليه بأفعال الكلام. }
\end{aligned}
$$

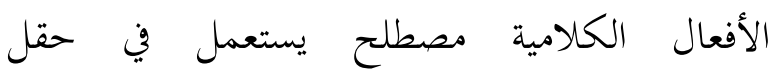

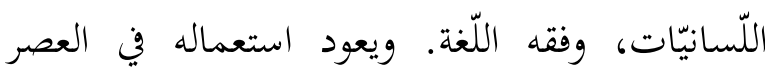

$$
\begin{aligned}
& \text { الحديث إلى الفيلسوف "جون لانجشو أوستن" اللّني } \\
& \text { قدّم للنّظريّة من خحلال بحوثه في الأفعال الكامية. } \\
& \text { وقد ميّز أوستن بين ثلاثة أنواع من الأفعال الكلامية، }
\end{aligned}
$$

\footnotetext{
${ }^{1}$ William P. Alston, Philosophy of Language (London: Prentice Hall Inc. London, 1964). H. 9
} 
إلى ذلك ، نجد دائمًا خمسة أفعال الكلام في المحادثات

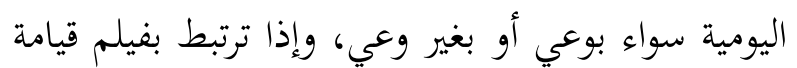
أرطغرل الحلقة الأولى يظهر فيه كثيرا من الأفعال

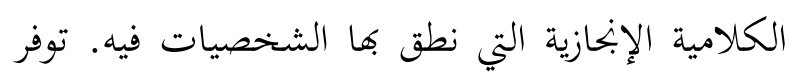

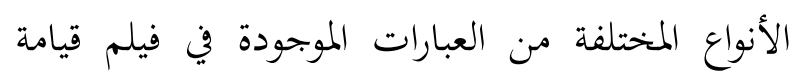
أرطغرل الحلقة الأولى للمخرج محمد بوزداغ ( Mehmet

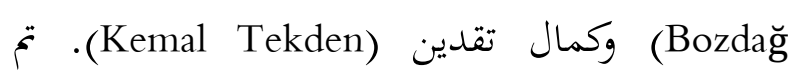

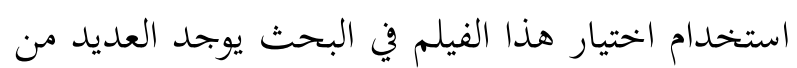
الحوارات المنطوقة التي تحتوي على الأفعال الكلامية الإنجازية. - المورات

بالإضافة إلى العديد من الأفعال الككلامية الإنحازية، يحتوي الفيلم أيضًا على قيمة جهاد أرطغرل

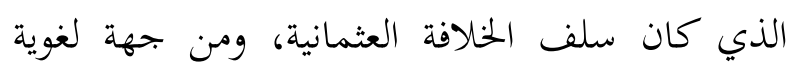

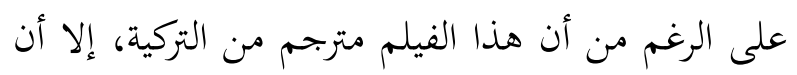

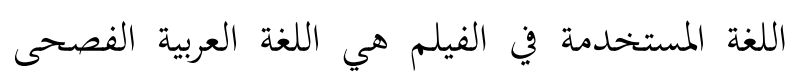

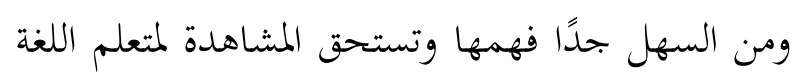
العبية اليوم لأن هناك كثير من القيمة اللغوية التي يمكن

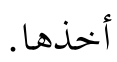

الإطار النظري

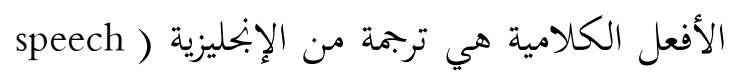

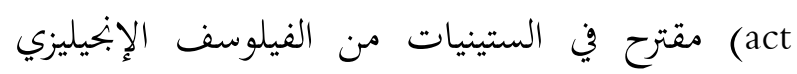

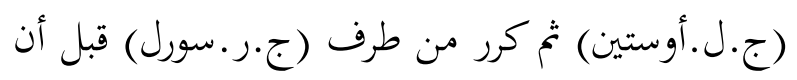

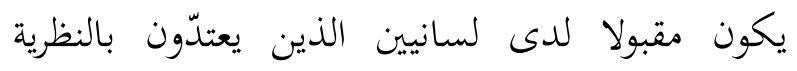

$$
\text { الملفوظة.6 - مقون }
$$

إن أول من يضع أساس نظرية الأفعال الكلامية هو أوستن سنة 1962 ثم يقوم بتطويرها

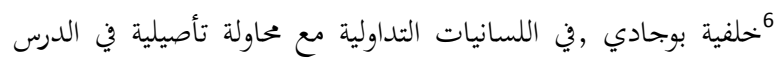

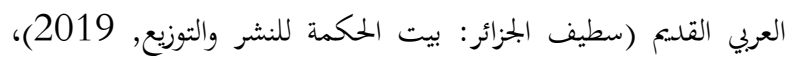
ص.
ينقلها كل لاعب فيلم. المحادثات الحوارية في الأفلام

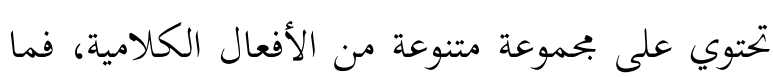

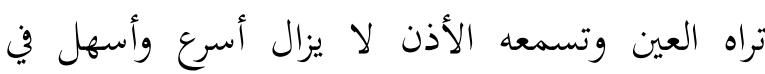
استيعاب العقل من قراءته التي تحتاج إلى الخيال للتعبير عنه.

الفيلم فن خيالي ناتج أساسًا عن أفكار

خيالية. ${ }^{3}$ يصدر حكمًا على أشخاص معينين. قالت نيا ديناتا

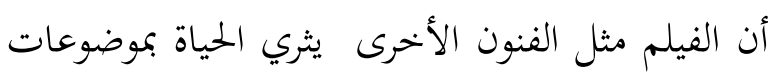

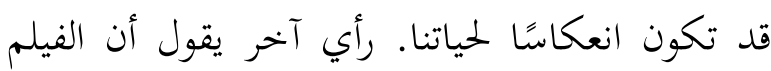

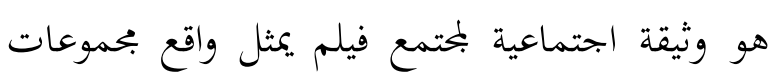

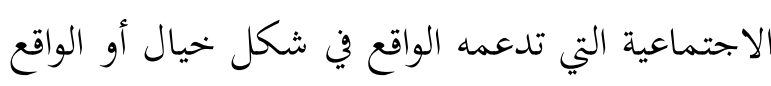
بمعناه الحقيقي. تم إجراء تحليل لأفعال الكلام من قبل الأخت غنى نور أولياه في السنة 2020 بالموضوع إنايل

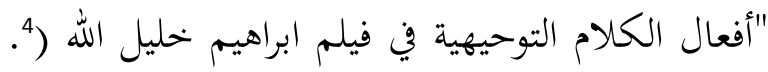

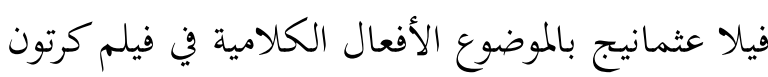

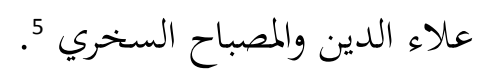
الأفعال الكلامية الإنحازية في حوار فيلم "قيامة

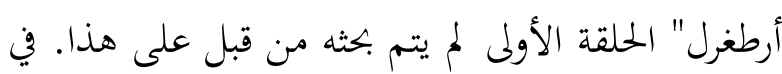

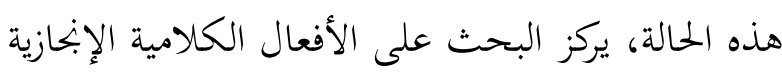

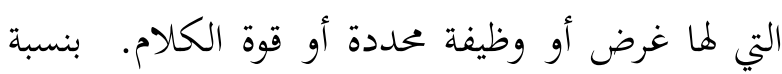
3عبد الحميد، العملية الإبداعية في فن التصوير (الكويت: عالم المعفرة، 1987)، ص. 66 أؤولياه, أنعال الكلام التوجيهية في فيلم إبراهيم خليل الله (كلية الأداب

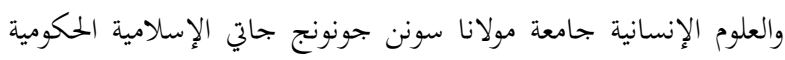

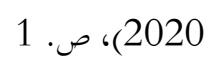

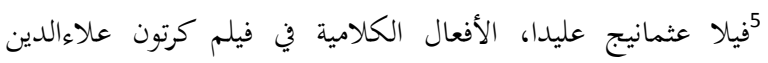

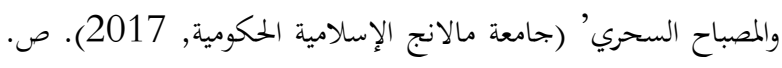


The act (الأول، الأفعال الإعلام أو قول شيء (s of saying something )، وهو الذي يسمى (s) بالأفعال اللفظية. والثاني، الأفعال التي تطلب من The act of doing ) المستمع لأن يفعل شيئ something )، وهي التي تسمى بالأفعال الإبحازية. ثم الثالث هو الأفعال التي تؤثر إلى المستمع، أو تؤدي إلى وجود رد فعل منه أو تأثير معين (The نتيجة من كلام (act of affecting someone المتكلم ، وهي التي تسمى بالأفعال التأثيرية9.

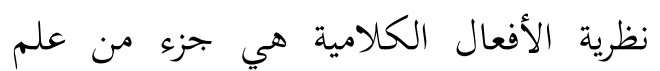
التداولي. إفها محاولة تعبير الجمل في الكلام كي يكون المستمع يفهم إرادة المتكلم. وهي عبارة تطلق كجزء من التفاعل الاجتماعي ${ }^{10}$ ـ الأفعال الكلامية جزء من الأحداث الكلامية، والأحداث الكلامية هي جزء من الحالات الكلامية. تقتصر كل الأحداث الكامية على الأنشطة التي تحكمها القواعد أو المبادئ الاجتماعية مباشرة للمتكلم. وبالتالي، فإن الفعل هو سمة من

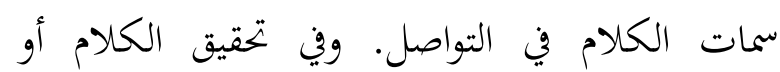
الخطاب يقوم الشخص بشيء، وهو أداء الفعل. الأفعال الكلامية هي عرض فردي ونفسي، وكانت استمراريتها تتعلق بالقدرة اللغوية للمتكلم في مواجهة أحوال معينة. يتم رؤيتها أكثر في معاني الأفعال عند الكالام. مناسبا بذلك، رآى سومارسونو أن الأفعال الكالامية هي عملية التعبير نتيجة للتفاعل الاجتماعي

${ }^{9}$ Austin. loc.cit.

${ }^{10}$ Abdul Chaer and Leonie Agustina, Sosiolinguistik Perkenalan Awal (Bandung: Rineka Cipta, 2010). H. 50

${ }^{11}$ Sumarsono, Sosiolinguistik (Jakarta: Pustaka Pelajar, 2017). H. 2
سريل سنة 1969. ذكر أوستن أنه عندما يقول الشخص شيئا، فإنه يفعل شيئا فإنه يفعل شيئا أيضا. أما يويلي فيقدم التعريف على أن الأفعال الكلامية هي

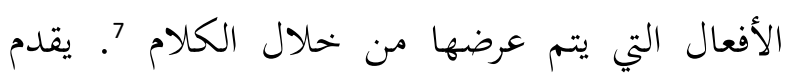
أوستن ثلاثة أنواع من الأفعال الكلامية، وهي الأفعال اللفظية (Lokusi)، والإبحازية (Ilokusi)، والتأثيرية Perlokusi). تحتوي الأفعال اللفظية على الكام

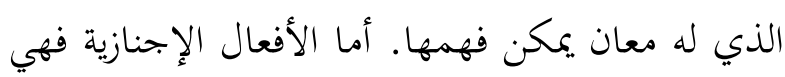
كل كلام يستخدم للقيام بالأعمال أو وظائف اللغة.

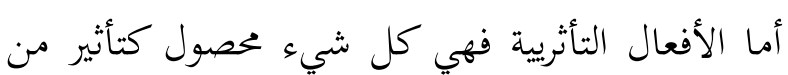
الكلام. ذكر سيرل أن المتكلم من منظور علم التداولي يمكن أن يحقق ثلاثة أنواع من الأفعال الكلامية وهي

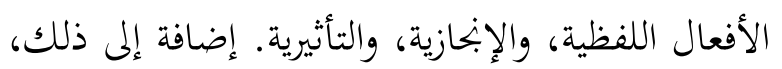
قال أن أنواع الأفعال الكلامية في كل لغة كانت متأثرة بالقوانين، و والقواعد، و والمعتقدات، و والعادة، والقيم الاجتماعية في الثقاف 8 .

الأفعال الكلامية هي الكلام الذي توجد فيه أفعال. عندما يقول المتكلم شيئا، كان يقوم

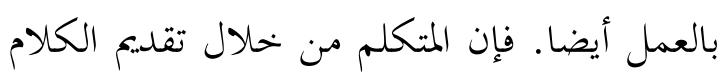
لديه هدف يريد أن يحققه من المستمع. هذا مناسب بما قاله أوستين (Austin) أن في القول عن شيء نحن نفعل شيئا أيضا". فهذا يعني أن المتكلم

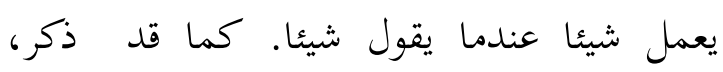
يقسم أوستن الأفعال الكلامية إلى ثلاثة أنواع:

7 Juha A. Vuori, 'Speech Act Theory', Research Methods in Critical Security Studies: An Introduction, 2013, 133-38 <https://doi.org/10.4324/9780203107119>.

${ }^{8}$ Napoleon M Mabaquiao, 'Speech Act Theory: From Austin to Searle', Augustinian Journal, 19.1 (2018), h. $1-18$. 
ميز سريل بين خمسة أنواع للأفعال الكلامية الإنحازية ولكل منها وظيفة تواصلية وهي الأفعال

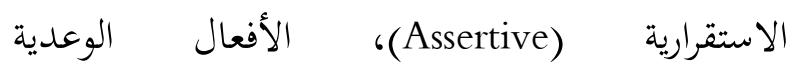

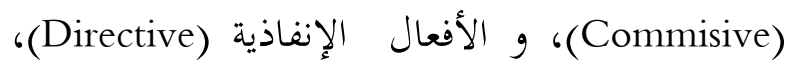
والأفعال التعبيرية (Expressive)، والأفعال التصريحية (Declarative)

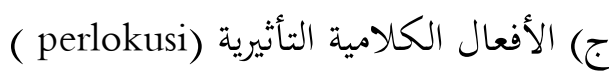

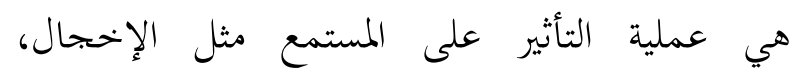
والتهايد، والمراودة، وغيرا. إنها نتيجة الكلام وتأثيره من الإنيان

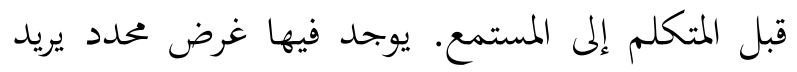
المتكلم رؤيته في أي رد. استنادًا إلى هذا البيان، فإن إن إنان

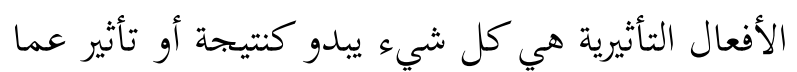

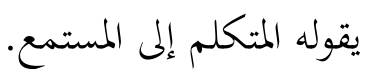

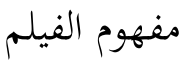

الفيلم أو الفلم (وجمعها أفلام) اصطلاحا هو فن أو مصنوعة ثقافية معمولة بواسطة ثقافات معينة والأفلام تعكس هذه الثقافات، وأيضا تؤثر فيها5.

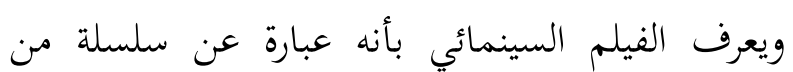

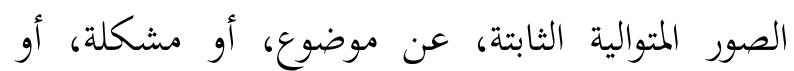
ظاهرة معينة، مطبوعة على شريط ملفوف على بكرة، حسب موضوعه والظروف التي تتاوح مدة عرضه عادة من عشرة دقائق إلى ساعتين، تحيط به. والأفلام السينمائية تعد وسيلة هامة من وسائل

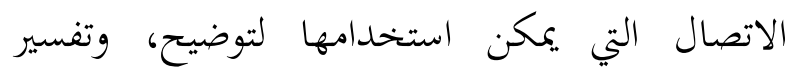

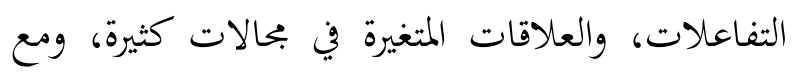
فئات وأعمار مختلفة؛ وتستخدم الأفلام السينمائية في

${ }^{14}$ K Rahardi, Pragmatik Kesantunan Imperatif Bahasa Indonesia (Jakarta: Erlangga, 2005). H. 36

15 منير البعلبكي ,قاموس المورد بيروت: دار العلم للملايين, 2014.
يقسم أوستن الأفعال الكلامية إلى ثلاثة أنواع،

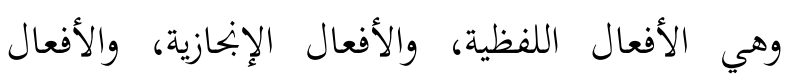

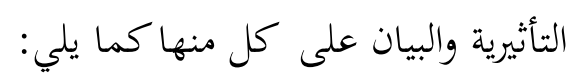
أ) الأفعال اللفظية (Lokusi)

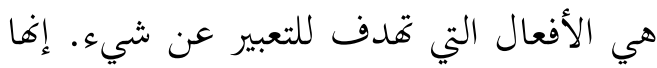

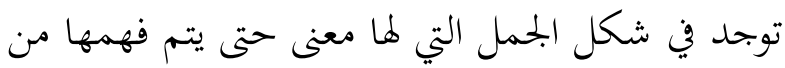

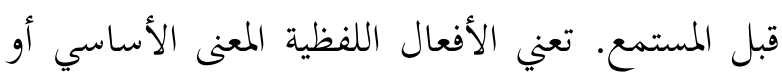

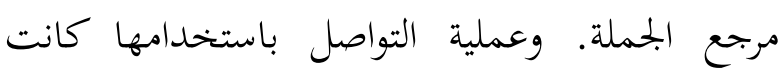
فكرية. فتفهم أن التعبيرات في الأفعال اللفظية تستخدم للتعبير عن شيء

بناءً على الفئة النحوية، بمكن تقسيم أشكال

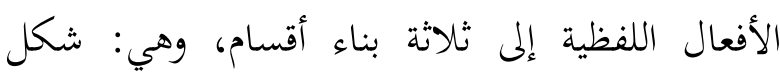

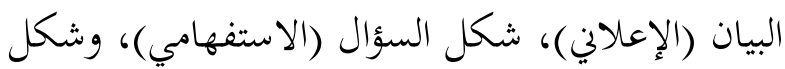

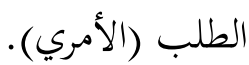
ب) الأفعال الإنجازية (Ilokusi) هي عملية تقديم البيانات، والأدعية، والوعود،

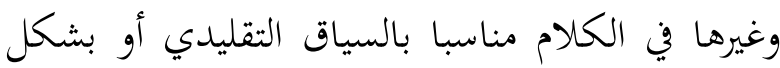

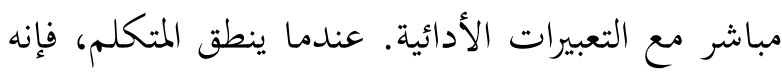

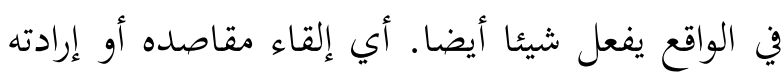

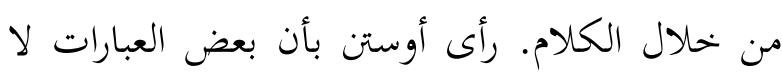

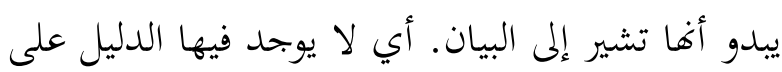

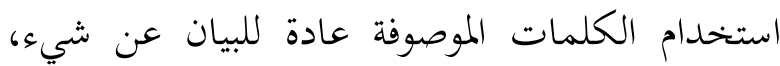

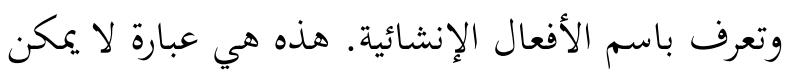
القياس أو المعرفة عن صحتها أو خطئها313

12 I Dewa Putu Wijana and Muhammad Rohmadi, Analisis Wacana Pragmatik Kajian Teori Dan Analisis (Surakarta: Yuma Pustaka, 2009). H. 17

James McCullough, 'Deborah Schiffrin. "Approaches to Discourse", Links \& Letters, 3, 1996, $147-49$. 
العداوة مع الصليبين ، وكان أحد الصليبيين الذين قتلوا هو الأخ الأصغر للقائد الصليبي تيتوس. ثم العائلة التي

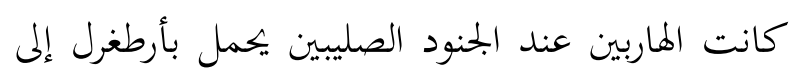
القبيلة كايي وتم اكتشاف أهم كانوا عائلة السلطان السلدوقي.

\section{منهجية البحث}

إن الطريقة المستخدمة في هذا البحث هي الطريقة الوصفية التحليلية ، ويتم جمع البيانات تبعا لهذه الطريقة

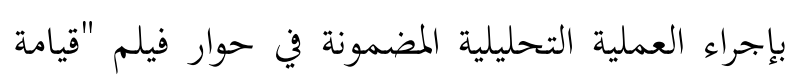

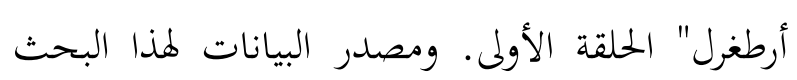
يعتمد على الدراسات الملكتبية. ومصصادر البيانات المستخدمة منها فيلم "قيامة أرطغرل" الحلقة الأولى التي تحتوي على الحوار من الأفعال الكلامية الإنحازية.
بحالات عديدة، ولأغراض متعددة حيث تستخدم في المجالات التعليمية، والإرشادية، والزراعية، والصناعية،

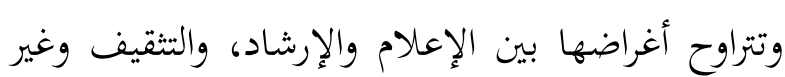

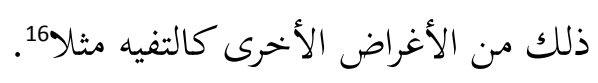
والفيلم بالموضوع "قيامة أرطغرل" أو بالتركية: هiriliş: Ertuğrul

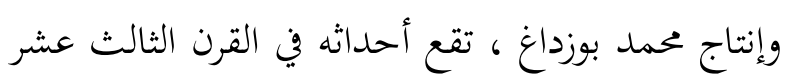
الميلادي، ويعرض مقدمات ودوافع تأسيس الدولة العثمانية من عرض سيرة حياة الغازي أرطغرل بن سليمان شاه، قائد قبيلة قايي من أتراك الأوغوز المسلمين ووالد عثمان الأول مؤسس الدولة العثمانية. وقام بدور أرطغرل الممثل التركي إبحين ألتان دوزياتان في 150 حلقة، وخمسة مواسم بعد أن شاهده نهو 3 مليارات مشاهد في العالم، وبث عبر شاشات في 71 دولة، مدبلجا إلى 25 لغة مختلفة، أبرزها العربية، والإبجليزية والأوردية.

كان أرطغرل ابن سليمان شاه، أحد زعماء القبائل في تركيا. سليمان شياه، زعيم القبيلة المتدين والتمسك بشدة التقاليد القبلية. في هذه البيئة ولد أرطغرل وكبر وتربي ليصبح بحاهد الإسلام. لدى أرطغرل

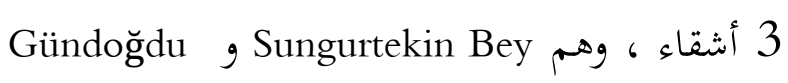

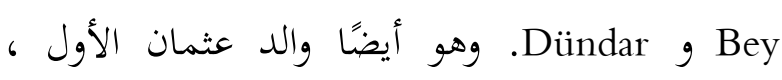
مؤسس الخلافة العثمانية. تبدأ القصة عندما يصطاد أرطغرل وثلاثة أصدقائه المخلصين وهم تورغوت وبامسي ودوغان في تبدان الغابة لكسب المخزون الغذائي لقبيلتهم. أثناء الصيد وديد ودئ

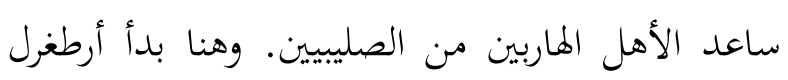

$$
\text { 16 الفيلم السينمائي أنواعه، وأهميته، وخصائصه, } 2007
$$


حوار فيلم "قيامة أرطغرل" الحلقة الأولى

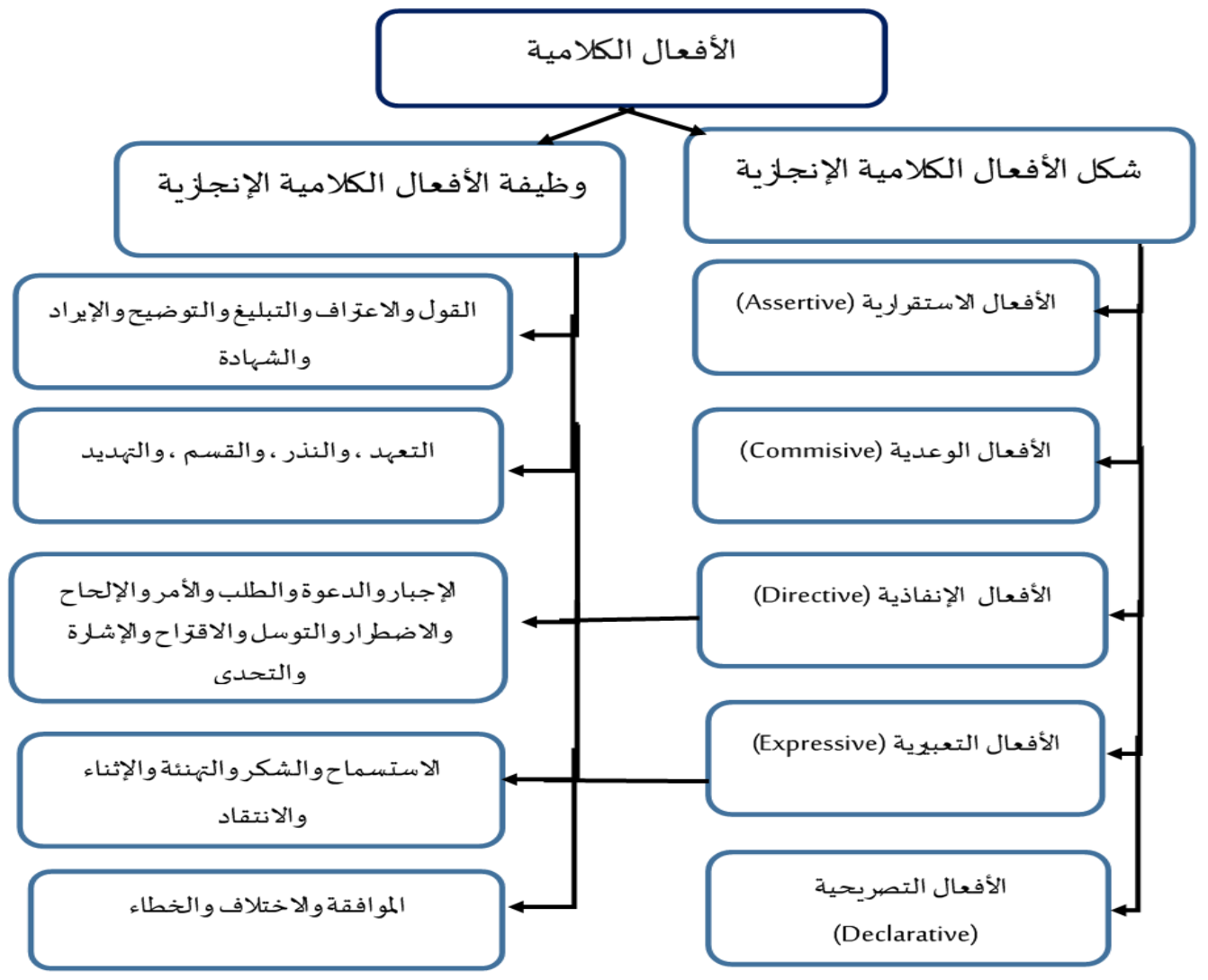

تحليلها ومناقشها

وقد تم جمع البيانات من خلال طريقة التدرج فيما

يتم البحث بوصف البيانات الموجودة في حوار فيلم يالي: (1) مشاهدة فيلم "قيامة أرطغرل" الحلقة الأولى، "قيامة أرطغرل" الحلقة الأولى. وتكون شكل الأفعال (2) استماع حوار فيلم "قيامة أرطغرل" الحلقة الأولى، الكلامية الإبحازية فيه أربعة : هي الأفعال الإستقرارية (3) كتابة حوار فيلم "قيامة أرطغرل" الحلقة الأولى، فيه 19 (Assertive)

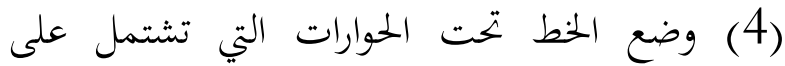
فيه 8 أفعال والأفعال الإفاذية (Commisive)

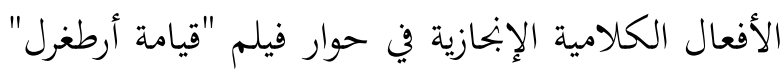
فيه 47 فعلا والأفعال التعبيرية (Directive) الحلقة الأولى. وقد تم تحليل البيانات بأسلوب التالي: فيه 15 فعلا والأفعال التصريكية (Expressive) (1) نقل البيانات التي تتعلق بالأفعال الكلامية الإبحازية (Declarative) في حوار فيلم "قيامة أرطغرل" الحلقة الأولى، (2) الجدول 1. الأفعال الكلامية الإبجازية في حوار فيلم

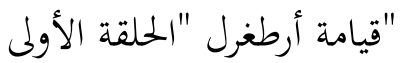
تصنيف البيانات حسب وظائفها باستخدام نظرية سريل، إيضاح البيانات من فوائد الأفعال الكلامية الإبجازية في حوار فيلم "قيامة أرطغرل" الحلقة الأولى ليل وشرحها إلى أشكال الأفعال الكلامية الإبجازية. 


\begin{tabular}{|c|c|c|}
\hline عداد & الوظيفة & الشكل \\
\hline 0 & الاعتراف & \multirow{5}{*}{$\begin{array}{c}\text { الأفعال الاستقرارية } \\
\text { (Assertive) }\end{array}$} \\
\hline 16 & التبليغ & \\
\hline 3 & التوضيح & \\
\hline 0 & الإيراد & \\
\hline 0 & الشهادة & \\
\hline 19 & & المجمو \\
\hline
\end{tabular}

\section{الأفعال الكلامية الإنجازية الوعدية}

هي فعل الكالام الذي يشجع المتكلم على هلى القيام بشيء. وبتحد الباحثة البيانات لهذا الفعل

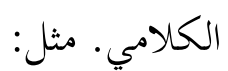
الحوار:

أرطغرل : "ما شاء الله، مسكين من سوف يقع تحته" صانع السيف : "فقط أنت تستحق هذا السيف، يجب أن يرحم من أجل حاق و نصر المظلوم إن شاء الله" الشرح : وظيفتها هي التعهد. وعد صانع السيف بأن ذلك السيف خاصا له ولن يستحق سواه لأنه يعتقد بإرتوجرول لشجاعته في مساعدة المظلومين. الجحدول 3. الأفعال الكلامية الإبجازية الوعدية

\begin{tabular}{|c|c|c|}
\hline البيانات & الوظيفة & الشكل \\
\hline 6 & التعهلد & \multirow{4}{*}{ الأفعال الوعدية } \\
\hline 0 & النذر & \\
\hline 0 & القسم & \\
\hline 2 & التهديد & \\
\hline 8 & \multicolumn{2}{|c|}{ المجموع } \\
\hline
\end{tabular}

\begin{tabular}{|c|c|c|c|}
\hline مئوية & علد البيانا & نوع العفل الكالامي & الرق \\
\hline$\% 21$ & 19 & $\begin{array}{c}\text { الأفعال الاستقرارية } \\
\text { (Assertive) }\end{array}$ & 1 \\
\hline$\% 9$ & 8 & الأفعال الوعدية & 2 \\
\hline$\% 53$ & 47 & الأفعال الإنفاذية & 3 \\
\hline$\% 17$ & 15 & $\begin{array}{l}\text { الأفعال التعبيرية } \\
\text { (Expressive) }\end{array}$ & 4 \\
\hline$\% 0$ & . & الأفعال التصريكية & 5 \\
\hline$\% 100$ & 89 & البحموع & \\
\hline
\end{tabular}

\section{الأفعال الكلامية الإنجازية الإستقرارية}

هي الفعل الكلام الذي تذكّر المتكلم بصدق ما يقال. وبتحد الباحثة البيانات لهذا الفعل الكلامي مثل: صانع السيف: "خيرا أرى أن رجالك يتضربون في

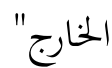
أرطغرل: "سنذهب لصيد وهم يتجهز" الشرح: وظيفتها هي التبليغ. يقول أرطغرل أن أصدقاءه الذي رأى صانع السيف كان يستعدون للصيد معه. الجدول 2. الأفعال الكلامية الإنحازية الإستقرارية 
صانع السيف: "نحمد الله أنه أرسلك إلينا يا بني"

أرطغرل: "حبا بالله وعلى شرف جدنا أوغثا... " الشرح: وظيفتها هي الشكر. شكر صانع السيف لله على إرسال أرطغرل إلى تلك القبيلة.

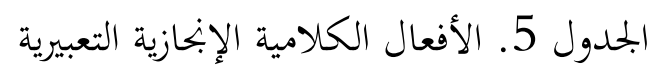

\begin{tabular}{|c|c|c|}
\hline عداد & الوظيفة & الشكل \\
\hline 0 & الاستسماح & \multirow{5}{*}{$\begin{array}{l}\text { الأفعال التعبيرية } \\
\text { (Expressive) }\end{array}$} \\
\hline 5 & الشكر & \\
\hline 4 & التهنئة & \\
\hline 6 & الثناء & \\
\hline 0 & الانتقاد & \\
\hline 15 & & البمحوِ \\
\hline
\end{tabular}

من عدد الفقرات، فإن أنواع الفعل الإنفاذي

هو نوع الفعل الكلامي الذي يظهر غالبا في حوار فيلم "قيامة أرطغرل" الحلقة الأولى. وهذا يدل على أن الفيلم "قيامة أرطغرل" هو فيلم تاريخي حرب حيث يكون

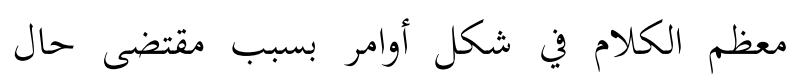
الحرب بحيث تكون وظيفة الأمر من الأفعال الإنفاذية مستخدم أكثر من الأفعال الأخرى في هذا الفيلم. مثل نتائج التحليل الذي أجراه الأخت فيلا عثمانيج عوليدا في السنة 2020 بالموضوع الأفعال الكلامية في فيلم كرتون علاء الدين والمصباح السخري.

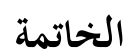

بناء على التحليل السابق في الباب الرابع

تستنبط الباحثة أن الأفعال الكامية الإبحازية في حوار

فيلم "قيامة أرطغرل" الحلقة الأولى تنقسم إلى أربعة :

هي الأفعال الإستقرارية (Assertive) فيه 19 فعلا

\section{الأفعال الكلامية الإنجازية الإنفاذية}

أي فعل الكلام الذي يعمل على تشجيع

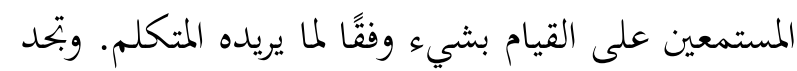
الباحثة البيانات لهذا الفعل الكامي كما يالي: الحوار : أرطغرل: "حتى الحديد ولم يحتمل بذكر الله"

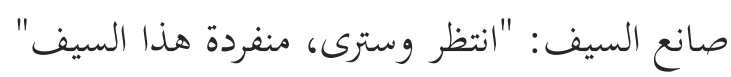
الشرح: وظيفتها هي الأمر. أمر سيد صانع السيف السريف أرطغرل بالانتظار لأنه أعد سيفا خاصا له. الجحدول 4. الأفعال الكلامية الإبحازية الانفاذية

\begin{tabular}{|c|c|c|}
\hline البيانات & الوظيفة & الشكل \\
\hline 1 & الإجبار & \multirow{10}{*}{ الأفعال الإنفاذية } \\
\hline 2 & الدعوة & \\
\hline 3 & الطلب & \\
\hline 30 & الأمر & \\
\hline 0 & الإلحاح & \\
\hline 2 & الاضطرار & \\
\hline 5 & التوسل & \\
\hline 3 & الاقتراح & \\
\hline 1 & الإشارة & \\
\hline 0 & التحدي & \\
\hline 47 & & البحموع \\
\hline
\end{tabular}
الأفعال الكالامية الإنجازية التعبيرية هي فعل الكاملام الذي يستخدم للتعبير عن الشعور والمواقف. وبتحد الباحثة البيانات لهذا الفعل بكل بكل

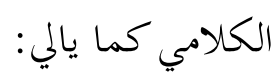
الحوار : 
<https://doi.org/10.4324/9780203107119 $>$

Wijana, I Dewa Putu, and Muhammad Rohmadi, Analisis Wacana Pragmatik Kajian Teori Dan Analisis (Surakarta: Yuma Pustaka, 2009)

Wiyatasari, Reny., 'Teknik Penerjemahan Tindak Tutur Direktif Dalam Cerpen Doktor Sihir Kaya Iwaya Sazanami Dan Larilah Melos Karya Dazai Osamu', Jurnal Bahasa Sastra Dan Budaya Jepang., 4, No.2 (2015), 46

أولياه, غنى نور, 'أفعال الكلام التوجيهية في فيلم

إبراهيم خليل الله' (جامعة مولانا سونن جونونج

$$
\text { جاتي الإسلامية الحكومية, 2020) }
$$

'الفيلم السينمائي أنواعه، وأهميته، وخصائصه ', (لهلى 2007

خلفية بوجادي, في اللسانيات التداولية مع محاولة تأصيلية في الدرس العربي القديم (سطيف الجزائر:

$$
\text { بيت الحكمة للنشر والتوزيع, 2019) }
$$

عبد الحميد, شاكر, العملية الإبداعية في فن التصوير (الكويت: عالم المعرفة, 1987)

عليدا, فيلا عثمانيج, "الأفعال الكلامية في فيلم كرتون لهال علاءالدين والمصباح السحري' (جامعة مالانج

$$
\text { الإسلامية الحلكومية, 2017) }
$$

مختار, درقاوي, 'نظرية أفعال الكلام "تعريف بالمافهم ',

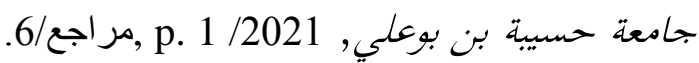

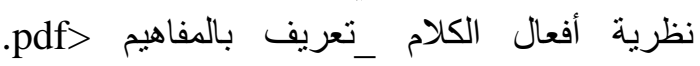
[accessed 30 July 2021]

منير البعلبكي, قاموس المورد (بيروت: دار العلم للمدايين, 2014)
والأفعال الوعدية (Commisive) فيه 8 فعلا والأفعال الإفاذية (Directive) فيه 47 فعلا والأفعال التعبيرية (Expressive) فيه 15 فعلا والأفعال التصريحية (Declarative) لا توجد.

تتكون الوظائف ل لأفعال الإستقرارية التي توجد في هذا البحث منها التبليغ (Assertive) بالعدد 16 فعلا والتوضيح بالعدد 3 أفعال. والوظائف للأفعال الوعدية Commisive) هي التعهد بالعدد 6 أفعال والتهديد فعلان. والوظائف للأفعال الإفاذية (Directive) هي الإجبار فعلا واحد والإشارة فعلا واحد والدعوة فعلان والاضطرار فعلان والطلب 3 أفعال والاقتراح 3 أفعال والتوسل 5 أفعال والأمر 30 فعلا. و والوظائف للأفعال التعبيرية هي الثناء 6 أفعال والشكر 5 أفعال (Expressive) والتهنئة 4 أفعال.

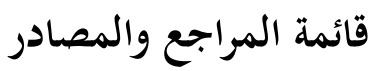

Alston, William P., Philosophy of Language (London: Prentice Hall Inc. London, 1964)

Austin, J. L, How to Do Things with Words (London: Oxford University Press, 1962)

Chaer, Abdul, and Leonie Agustina, Sosiolinguistik Perkenalan Awal (Bandung: Rineka Cipta, 2010)

Mabaquiao, Napoleon M, 'Speech Act Theory: From Austin to Searle', Augustinian Journal, 19.1 (2018), 1-18

McCullough, James, 'Deborah Schiffrin. "Approaches to Discourse", Links \& Letters, 3, 1996, 147-49

Rahardi, K, Pragmatik Kesantunan Imperatif Bahasa Indonesia (Jakarta: Erlangga, 2005)

Sumarsono, Sosiolinguistik (Jakarta: Pustaka Pelajar, 2017)

Vuori, Juha A., 'Speech Act Theory', Research Methods in Critical Security Studies: An Introduction, 2013, 133-38 